\title{
An examination of Professor Shaikh's proposal to tame Harrodian instability
}

\author{
Reiner Franke* \\ University of Kiel, Germany
}

This note reconsiders a recent proposal by A. Shaikh to tame Harrodian instability (Metroeconomica 2009), where besides the utilization gap investment depends on the expected growth of demand. His stability result has, however, been criticized as not credible. The crucial point is that Shaikh's continuous-time treatment does not distinguish between forward and backward derivatives. In order to check whether or not this poses a problem, several slight modifications of the model in continuous and discrete time are formulated and investigated for their stability. Roughly speaking, it is found that unless one assumes (myopic) perfect foresight, the destabilizing Harrodian mechanism continues to be effective.

Keywords: Hicks-Harrod adjustment principle, continuous-time versus discrete-time modelling, backward-looking versus forward-looking expectations

JEL codes: $C 02, D 84, E 12, E 30$

\section{INTRODUCTION}

The frame of reference of this contribution is the Kaleckian model of growth and distribution in its simplest form with a savings and investment function, IS market clearing, and one dynamic mechanism that moves the economy from the short run to the long run. More specifically, it is concerned with the strand of the theory that conceives of the normal rate of capacity utilization as a given target. In this way, however, the problem of Harrodian instability arises, a process of cumulative causation where an excess of actual over normal utilization drives up the growth rate of fixed capital, which via the multiplier increases utilization, which in turn further boosts investment, etc. On the other hand, theorists following this approach do not view the economy as being globally unstable. Thus, they face the challenge of devising an additional mechanism that may tame the destabilizing Harrodian forces.

One such mechanism has recently been proposed by Shaikh (2009). He assumes that, apart from the usual response to the utilization gap, investment is based on the expected growth of sales. Within the continuous-time framework in which this is formulated, the goods market clearing and a simple mathematical operation bring about an exact reversal of the Harrodian reactions. Unfortunately, the price for this solution is doubts about the economic meaning of the dynamic process.

Summarizing the assessment by Hein et al. (2011: 605-608), the critical issue is the interpretation of the expectations, which are not unambiguous in continuous time. The

* Email: franke@uni-bremen.de. I wish to thank Peter Flaschel and Marc Lavoie for fruitful remarks.

Received 22 August 2014, accepted 25 September 2014 
process makes sense if they are rationally formed - which in the eyes of most non-orthodox economists would demand too much from the firms in the model. ${ }^{1}$ If, by contrast, firms more reasonably behave in an adaptive manner, the dynamic adjustments are 'not credible at all' (Hein et al. 2011: 608). Nevertheless, as informative as it is, a problem with this discussion is that it remains at an informal level, so that the contradiction between credibility and a definite mathematical stability result is not fully understood.

In the course of Shaikh's analysis, an equation is obtained that contains a forward and a backward derivative, which then in a purely technical operation cancel out. This is something one may feel uneasy about right at the beginning, as it prompts the question of what would become of the dynamics in an explicit discrete-time setting. For this purpose the economy is formulated with an adjustment (or market) period of positive length $h>0$. The concept of such an $h$-economy, suitably set up, allows us to ask whether in the limit for $h \rightarrow 0$ the original model in continuous time would be recovered. If the model does not pass this consistency check we can still ask for the stability of the $h$-economies. As will be seen, the answers to these question depend on the precise specification of the kind of expectations, especially whether they are 'forward-looking' or 'backward-looking'. Our aim is that in this way the basic insights by Hein et al. (2011) are given a more rigorous basis.

The remainder of the paper is organized as follows. Section 2 reiterates Shaikh's proposal and his stability argument. Section 3 formulates the economy in discrete time with a market period of flexible length. This setting allows us to distinguish between three dating specifications in the firms' sales expectations and to analyse their implications for stability. Section 4 introduces the more appropriate concept of adaptive expectations and examines them in both a continuous-time and discrete-time framework. Section 5 concludes.

\section{PROFESSOR SHAIKH'S FORMULATION}

Our point of departure is the standard Kaleckian model as it is laid out by, for example, Hein et al. (2011: section 2). It considers a closed one-good economy without taxation, government spending, capital depreciation and inflation. Rather than refer to capacity utilization, as these authors do, we directly use the output-capital ratio $u=Y / K$ to measure economic activity. It is the utilization rate of the capital stock in place, or just 'utilization' for short. Labour being in perfectly elastic supply, the IS part of the model is constituted by the following three equations:

$$
\begin{aligned}
& g^{s}=s r=s \pi u \\
& g_{k}^{i}=a+\gamma\left(u-u_{n}\right) \\
& g_{k}^{i}=g^{s} .
\end{aligned}
$$

The function $g^{s}$ in the first equation represents the savings in the economy normalized by the (replacement value of the) capital stock: workers consume all of their wages and $s$ is the capitalists' propensity to save out of their profits. Correspondingly, $r$ is the rate of profit and $\pi$ the share of profits in total income. Both $s$ and $\pi$ are supposed to be constant.

Equation (2) specifies the investment function; that is, the planned growth rate of the capital stock. Generally speaking, it is based on a benchmark rate of growth $a$, where the

1. In fact, in an early remark on Shaikh's proposal soon after it was first presented in a working paper, Skott (2008: 22, fn 28) directly interpreted the dynamic process as being 'based on the assumption of fulfilled expectations at all times' (see Skott 2010: 127, fn 28, for the published version of Skott 2008). 
symbol ' $a$ ' is chosen since this rate is occasionally referred to as the firms' 'animal spirits'. With a reaction coefficient $\gamma>0$, the second component of investment says that in the presence of overutilization, when actual utilization $u$ exceeds the so-called normal rate of utilization $u_{n}$, the firms seek to close this gap relatively fast by increasing the capital stock at a higher rate than $a$; and analogously for underutilization. Normal utilization is treated as an exogenous and fixed target.

The third equation postulates temporary equilibrium on the goods market, where market clearing is brought about by quantity variations. Solving (3) for $u$, utilization in this state can be written as:

$$
u=\frac{a-\gamma u_{n}}{s \pi-\gamma}
$$

The denominator is assumed to be positive. This is nothing other than the well-known Keynesian stability condition, which we will not call into question here. A steady-state position (denoted by a superscript ' $o$ ') is given by normal utilization, $u^{o}=u_{n}$, and a growth rate of output and the capital stock $g^{o}=a^{o}=s \pi u_{n}$.

Shaikh's general idea is that firms adopt the expected growth rate of sales as their benchmark $a$. Under the assumption of IS, it can be identified with the expected output growth. Before turning to a formal specification, we can try to argue in an informal way. To this end, let us undertake the common exercise that, being in a long-run equilibrium, the economy experiences a (once-and-for-all) decline in the savings propensity $s$ (which incidentally lowers the steady-state growth rate). If there is no immediate reaction in the sales expectations, the first change is an increase of utilization in (4) above normal. This means that output and sales rise faster than before. If the sales expectations take this observation into account in some adaptive manner, the benchmark growth rate will increase, too. As a consequence, $u$ increases further, which accelerates output growth, which in turn should lead to a further rise in the expected growth of sales, etc. So far, this is just the standard Harrodian line of reasoning and one may wonder what difference in a formal model it should make if, instead of specifying the rate of change of $a$ as a function of the utilization gap $\left(u-u_{n}\right)$ (as, for example, in the treatment by Hein et al. 2011: 592), this benchmark is conceived of as the expected growth of sales.

In his proposal, Shaikh (2009: 465) starts out from the investment function and formulates his counterpart of equation (2) directly with the output growth rate $g_{y}$ as the benchmark:

$$
g_{k}^{i}=g_{y}+\gamma\left(u-u_{n}\right)
$$

As he works in continuous time, he need not say a word about possible information lags in this relationship. Referring to Hicks (1985: 97-107), Shaikh calls (5) the Hicks-Harrod $(\mathrm{HH})$ dynamic adjustment principle. With the usual notation $\dot{x}=$ $d x / d t$ and $\hat{x}=\dot{x} / x$ for a dynamic variable $x=x(t)$, logarithmic differentiation of the identity $Y=u K$ gives $g_{y}=\hat{u}+\hat{K}=\hat{u}+g_{k}$. Because by virtue of IS all investment plans can be realized, $g_{k}^{i}=g_{k}$, (5) can be rewritten as:

$$
g_{k}=\hat{u}+g_{k}+\gamma\left(u-u_{n}\right) .
$$


It only remains to let the capital growth rates cancel out and rearrange the terms, and Shaikh can conclude that equation (5) is equivalent to:

$$
\hat{u}=-\gamma\left(u-u_{n}\right)
$$

According to this differential equation, 'the $\mathrm{HH}$ adjustment process is completely stable around $u=u_{n}$ (the warranted path)' (Shaikh 2009: 465). In other words, the Hicks-Harrod investment function (5) is capable of solving the problem of Harrodian instability.

Now, in the light of the straightforward Harrodian argument above, one might appreciate the elementary mathematical procedure or feel it is in the end no more than a sleight of hand. To prepare the ground for a critical discussion, it is helpful to think about how precisely equation (5) would read in a discrete-time setting. After all, the story of the dynamic feedbacks that we told was cast in discrete steps, too. As the left-hand side of (5) is fixed investment in the time interval $[t, t+1)$, the capital growth rate $g_{k}^{i}$ means $\left(K_{t+1}-K_{t}\right) / K_{t}$. What, then, is the dating of the output growth rate on the right-hand side? If expectations, as in our story, are adaptive, $g_{y}$ is the most recent observation of output growth; that is, it is equal to $\left(Y_{t}-Y_{t-1}\right) / Y_{t-1}$, or even $\left(Y_{t-1}-Y_{t-2}\right) / Y_{t-2}$. Accordingly, the capital growth rate on the right-hand side in (6) is $\left(K_{t}-K_{t-1}\right) / K_{t-1}$, or even $\left(K_{t-1}-K_{t-2}\right) / K_{t-2}$. Hence the two capital growth rates in a discrete-time counterpart of (6) would be differently dated and would not simply cancel.

For the continuous-time formulation, this observation means that $g_{k}$ on the left-hand side of (6) represents a forward derivative $\dot{K}^{+} / K:=\lim _{h \rightarrow 0}[K(t+h)-K(t)] / h K(t)$, whereas on the right-hand side we have a backward derivative $\dot{K}^{-} / K:=$ $\lim _{h \rightarrow 0}[K(t)-K(t-h)] / h K(t-h)$. The transition from (6) to (7) then assumes that the two limits coincide. If this assumption is accepted - and why should it not? - we seem to have a contradiction between an obvious process of cumulative causation yielding instability and a hard mathematical stability result.

Regarding the discrete-time scenario it may also be remarked that the previous argument would be undermined if the supposition of adaptive, or 'backward-looking', expectations is abandoned and instead perfect foresight is stipulated, $g_{y}=\left(Y_{t+1}-Y_{t}\right) / Y_{t}$ in (5). It might be conjectured that this is a case that is compatible with Shaikh's mathematical argument.

What we infer from these reflections is that it should indeed be useful to put Shaikh's idea in perspective by reconsidering it in a discrete-time framework. In particular, it will force us to make explicit reference to the kind of expectations in the investment function, whether they are forward-looking or backward-looking. In the next section we want to make these issues more precise. Specifically, proper discrete-time modelling should allow for a flexible adjustment period, so that we can also ask what would happen in the limit when it becomes arbitrarily short.

\section{THE HICKS-HARROD MECHANISM IN DISCRETE TIME}

With respect to a given unit of time (a year, say), we slice time into intervals of length $h>0$; that is, $[t-h, t),[t, t+h)$, etc., where $h$ may be as small as we want. These periods will be our adjustment periods, or market periods, and the corresponding economies may occasionally be referred to as $h$-economies. $K_{t}$ is the capital stock in place at the beginning of the period $[t, t+h) . Y_{t}$ is the 'annualized' rate of output in the same period, so that the 
total volume of output over $[t, t+h)$ is $h Y_{t}$. Certainly, capital utilization is $u_{t}=Y_{t} / K_{t}$. The (likewise annualized) growth rates of these variables are all defined backwards:

$$
g_{x, t}:=\frac{x_{t}-x_{t-h}}{h x_{t-h}}(x=k, y), \quad \hat{u}_{t}:=\frac{u_{t}-u_{t-h}}{h u_{t-h}}
$$

(reference to the underlying length of the market period is omitted in this notation as it will be understood from the context). With $K_{t} / K_{t-h}=1+h g_{k, t}$ and $1+h g_{y, t}=1+\left(Y_{t}-Y_{t-h}\right) / Y_{t-h}=Y_{t} / Y_{t-h}=\left(Y_{t} / K_{t-h}\right) /\left(Y_{t-h} / K_{t-h}\right), \quad$ it is easily checked that the output growth rate can alternatively be expressed in the following ways:

$$
\begin{aligned}
& g_{y, t-j h}=\frac{1}{h}\left[\frac{u_{t-j h}}{u_{t-(j+1) b}}\left(1+h g_{k, t-j h}\right)-1\right], \quad j=1,0,-1 \\
& g_{y, t-j h}=\hat{u}_{t-j h}+g_{k, t-j h}+h \hat{u}_{t-j h} g_{k, t-j b} .
\end{aligned}
$$

These relationships will be helpful in the analysis below. Regarding the sales expectations that the firms have to form when at the beginning of the period $[t, t+b)$ they decide on investment, three cases may be distinguished. First, the firms have rational expectations, so they already know output and sales over the next period and thus the output growth rate $g_{y, t+h}$. Second, they know what will be produced and sold over the period now to evolve. Accordingly, they know $g_{y, t}$, which represents the case of myopic perfect foresight. Third, the only information firms can use is what they have observed in the previous period $[t-h, t)$. In line with the specification above, they base their investment directly on the previous growth rate $g_{y, t-h}$; that is, they entertain static expectations. Thus, we have the following three alternative dating assumptions in the Hicks-Harrod investment function (5).

Rational expectations:

$$
g_{k, t+h}^{i}=g_{y, t+h}+\gamma\left(u_{t}-u_{n}\right)
$$

Myopic perfect foresight:

$$
g_{k, t+h}^{i}=g_{y, t}+\gamma\left(u_{t-h}-u_{n}\right)
$$

Static expectations:

$$
g_{k, t+h}^{i}=g_{y, t-h}+\gamma\left(u_{t-h}-u_{n}\right)
$$

Annualized savings in period $[t, t+h)$ amount to $s \pi Y_{t}$. Dividing by $K_{t}$ and writing $g_{t+h}^{s}$ in conformity to the notation of the investment function, the savings function is for all three cases the same:

$$
g_{t+h}^{s}=s \pi u_{t}
$$

Of course, in this setting the IS equilibrium condition (3) for period $[t, t+b)$ refers to $g_{k, t+h}^{i}$ and $g_{t+h}^{s}$. Let us then consider each of the three assumptions on the sales expectations in turn. 


\subsection{Rational expectations}

Substituting the rational expectation assumption and then identity (10) with $j=-1$ in the market clearing condition $g_{t+h}^{s}=g_{k, t+h}^{i}$ gives us the relationship $s \pi u_{t}=\hat{u}_{t+h}+g_{k, t+h}+h \hat{u}_{t+h} g_{k, t+h}+\gamma\left(u_{t}-u_{n}\right)$. The capital growth rate equals $g_{t+h}^{s}=s \pi u_{t}$, upon which by virtue of (11) the saving terms $s \pi u_{t}$ on the left-hand and right-hand sides cancel out. Solving for $\hat{u}_{t+h}$ leads to

$$
\hat{u}_{t+h}=\frac{-\gamma\left(u_{t}-u_{n}\right)}{1+h s \pi u_{t}} .
$$

This is the counterpart of Shaikh's equation (7) and the continuous-time limit $h \rightarrow 0$ is well-defined, too. For the dynamic equation (12) itself, the steady state is stable for all values of $h>0 .^{2}$ Hence everything is well - and Shaikh's stability conclusion as well as the abovementioned insight by Hein et al. (2011) are fully confirmed - if one is willing to accept the hypothesis of rational expectations.

\subsection{Myopic perfect foresight}

The same procedure as before now gives us $s \pi u_{t}=\hat{u}_{t}+s \pi u_{t-h}+h \hat{u}_{t} s \pi u_{t-h}+\gamma\left(u_{t-h}-u_{n}\right)$. Observing that $s \pi\left(u_{t}-u_{t-h}\right)=h s \pi u_{t-h}\left(u_{t}-u_{t-h}\right) / h u_{t-h}=h s \pi u_{t-h} \hat{u}_{t}$, which subsequently cancels out, the equation becomes $\hat{u}_{t}=-\gamma\left(u_{t-h}-u_{n}\right)$. For a comparison with (12) it may also be dated forward:

$$
\hat{u}_{t+h}=-\gamma\left(u_{t}-u_{n}\right) .
$$

Convergence towards normal utilization is again ensured - even at a lower price than before, as instead of (full) perfect foresight, myopic perfect foresight already proves sufficient. This is a conclusion that in this precise form is not available from the geometric and verbal discussion by Hein et al. (2011). ${ }^{3}$

How, then, may we assess the assumption of myopic perfect foresight: is it not at the same level of abstraction as the market clearing condition? On the basis of our discretetime story, the question has to be negated. Recall that firms decide on investment at the beginning of the period $[t, t+b)$, and it is then that they also have to form their expectations about the sales, or the output, over this period. Consumption and production, however, develop only subsequently, and it is a process of mutual adjustments then setting-in that is supposed to be so effective that at the end of the period total production happens to have matched aggregate demand (which does not rule out that individual firms will have produced too much or too little). Hence the myopic perfect foresight of sales requires capabilities of acquiring and processing information from the firms that cannot be too easily taken for granted.

2. Rearrange (12) such as to obtain a difference equation $u_{t+h}=f\left(u_{t}\right)$ for a suitable function $f(\cdot)$, consider the stability condition $\left|d f\left(u_{n}\right) / d t\right|<1$, establish that it is tantamount to bү $u_{n} /\left(1+h s \pi u_{n}\right)=\gamma u_{n} /\left(1 / h+s \pi u_{n}\right)<2$, and note that by virtue of the Keynesian stability condition $\gamma<s \pi$ the left-hand side is always less than one.

3. To be exact, stability in (13) requires that $h$ be not too large as the argument sketched in the previous footnote now gives $h<2 / \gamma u_{n}$. 


\subsection{Static expectations}

Under static (or naive) expectations, everything is predetermined in the investment function, and the IS condition together with (11) determines utilization $u_{t}$ directly from past data, $u_{t}=(1 / s \pi)\left[g_{y, t-h}+\gamma\left(u_{t-h}-u_{n}\right)\right]$. Incidentally, we do not even need to be concerned about Keynesian stability; $\gamma$ may or may not be less than $s \pi$. It remains to use equation (9) with $j=1$ and replace $g_{k, t-h}$ in it with $g_{t-h}^{s}=s \pi u_{t-2 h}$ from a redating of (11). In this way a second-order difference equation in utilization is obtained:

$$
\begin{aligned}
u_{t} & =f\left(u_{t-h}, u_{t-2 h}\right) \\
& :=\frac{1}{s \pi}\left\{\frac{1}{h}\left[\frac{u_{t-h}}{u_{t-2 h}}\left(1+h s \pi u_{t-2 h}\right)-1\right]+\gamma\left(u_{t-h}-u_{n}\right)\right\}
\end{aligned}
$$

It may be noted that if, in the argument leading to (14), one uses identity (10) instead of (9) for the lagged output growth rate, a difference quotient could show up again. Unfortunately, with $u_{t}$ already present in the relationship, it would be of no use, as it is backward-looking, $\hat{u}_{t-h}=\left(u_{t-h}-u_{t-2 h}\right) / h u_{t-2 h}$, and so cannot drive the system forward.

On the other hand, we could subtract $u_{t-h}$ on both sides of (14) and divide the equation by $h$. In this case, however, the absolute value of the right-hand side would generally grow without bounds as the length of the adjustment period tends to zero, $h \rightarrow 0$. In other words, the $h$-economies with static expectations cannot converge towards Shaikh's differential equation (7), simply because they do not have a well-defined continuous-time limit at all.

The latter feature might even lead to the assessment that, in the desire for simplicity, something is inexpedient in the specifications underlying equation (14). This judgement is based on the methodological postulate that every model formulated in discrete time should remain well-defined as its adjustment period shrinks to zero (a postulate one may infer from Foley 1975, a once-influential paper). We nevertheless leave such a consistency requirement here as an open issue.

The non-existing continuous-time limit notwithstanding, the local stability of the discrete-time system can be examined for any arbitrary value of $h$. Of course, $f\left(u_{n}, u_{n}\right)=u_{n}$, so that normal utilization is indeed a fixed-point of (14) for all $h$. Putting $v_{t}=u_{t-h}$ and writing the difference equation as

$$
\left[\begin{array}{l}
u_{t} \\
v_{t}
\end{array}\right]=\left[\begin{array}{c}
f\left(u_{t-h}, v_{t-h}\right) \\
u_{t-h}
\end{array}\right]
$$

allows us to set up the Jacobian matrix $J$ (evaluated at $u_{n}$ ) of this (non-linear) twodimensional system and refer to the Schur conditions for its stability. They are based on the coefficients

$$
\begin{aligned}
& a_{1}:=-\operatorname{trace} J=-\partial f / \partial u_{t-h}=-1-\frac{\gamma}{s \pi}-\frac{1}{h s \pi u_{n}} \\
& a_{2}:=\operatorname{det} J=-\partial f / \partial u_{t-2 h}=\frac{1}{h s \pi u_{n}} .
\end{aligned}
$$

One of the necessary conditions for stability is $1+a_{1}+a_{2}>0$ (see, for example, Gandolfo 1997: 58). However, what we get is $1+a_{1}+a_{2}=-\gamma / s \pi$. Hence with static expectations the discrete-time economies are all unstable, however short their market 
period $h$ may be. We have thus provided an explicit framework to decide about the stability issue of the Hicks-Harrod principle. It rigorously confirms the more informal reasoning by Hein et al. (2011: 605-608) that, at least with the simple backward-looking sales expectations conforming to Shaikh's growth-rate specification, this mechanism is not able to overcome the original Harrodian instability in the economy.

\section{ADAPTIVE EXPECTATIONS}

Interestingly, in the exposition of his model, Shaikh (2009: 465) also adds random shocks to the functional relationships. ${ }^{4}$ Such a noisy environment draws attention to the fact that in forming their expectations the firms may not want to react with full strength to each and every sudden jump in the shock process, especially when the resulting investment is irreversible. A straightforward expectations hypothesis that allows for smoother forecasts is adaptive expectations (AE). In the wake of the New Classical revolution they have been criticized as rather foolish because of their systematic prediction errors. Nonetheless, Flaschel et al. (1997: chs 7.2 and 7.3) discuss how, apart from their non-negligible empirical significance, $\mathrm{AE}$ can be more reasonable than they might appear at first sight (one aspect being that in a stochastic setting, even if there are no structural breaks, it will take a long time to reliably detect a serial correlation in the prediction errors).

In particular, adaptive expectations can be interpreted as approximately representing the one-period-ahead extrapolative forecasts that are obtained from linear regressions of a variable upon time with a constant rolling sample period (ibid.: 158), which is a natural device for firms that seek to behave like econometricians. From a slightly different angle, $\mathrm{AE}$ can also be viewed as a subcase of recursive least-squares learning, namely constant gain (or perpetual) learning, which under certain conditions even has some optimality properties and is widely used in the recent learning literature. ${ }^{5}$ Constant gain learning is more general than $\mathrm{AE}$ in that the speed of adjustment (corresponding to our coefficient $\beta$ below) is endogenously determined from past observations and is thus varying over time. Not too rarely, however, this speed is directly treated as fixed. ${ }^{6}$ Thus, somewhat ironically, adaptive expectations have experienced a certain rehabilitation, only that they now feature under the name of constant gain learning.

These few remarks may suffice to indicate that adaptive expectations are a perspective worth studying as a next simple step in the analysis, a concept that seeks to avoid the idea that too much takes place simultaneously. Besides, in the discrete-time framework AE include the static expectations from above as a special case, as will be easily seen later. Before that, we consider their modelling in continuous time.

\subsection{A continuous-time approach}

Under the assumption of adaptive expectations, the sales expectations of the firms are a predetermined variable, captured by the benchmark rate of growth $a$ introduced in

4. Although it is not clear how his $\varepsilon$-term is supposed to apply in the differential equation for $u$ (equation (7) in the present paper), where in a mathematically correct but elaborated way the perturbations may have to be represented by something like a Wiener process.

5. Two standard references are Sargent (1999) and Evans/Honkapohja (2001).

6. One example is Cellarier (2006: 59), but there are many more. 
Section 2. This rate is gradually increased (decreased) when the most recent output growth is above (below) it. Thus in continuous time the principle reads:

$$
\dot{a}=\beta\left(g_{y}-a\right) .
$$

A usual view on this equation is that an infinite adjustment speed, $\beta=\infty$, is tantamount to the case of myopic perfect foresight, $a=g_{y}$, with which we would return to Shaikh's specification.

As a first consequence of (15), IS utilization is determined by equation (4), $u=u(a)=$ $\left(a-\gamma u_{n}\right) /(s \pi-\gamma)$ with Keynesian stability presupposed $(\gamma<s \pi)$. A common-sense reasoning for understanding the dynamics induced by this type of expectation can be very similar to the one discussed in Section 2. So far, it is not apparent why AE may have a soothing effect on the destabilizing Harrodian forces.

Turning to the mathematics, we can come back to Shaikh's treatment and express the output growth rate as $g_{y}=\hat{u}+g_{k}$. With (4), we now have $\hat{u}=\dot{a} /\left(a-\gamma u_{n}\right)$, while for the capital growth rate we may once again make use of $g_{k}=g^{s}=s \pi u$. Plugging this into (15), the following relationship is obtained:

$$
\dot{a}=\beta\left[\frac{\dot{a}}{a-\gamma u_{n}}+s \pi u(a)-a\right] .
$$

From the discussions above we should be sensitive enough to notice that the first derivative in (15) is a forward derivative, which determines the benchmark rate $a$ in the 'next' (infinitesimally short) period. By contrast, the growth rate $g_{y}$ on the right-hand side of the equation refers to what firms were able to observe in the recent past. Correspondingly, the rate of change in utilization in the derivation of (16) is a backward derivative. Again, with (16) we have a relationship where one of the derivatives is forward and the other backward, something that we will have to take more explicitly into account in the discretetime respecification further below.

In the present subsection we assume that we are permitted to treat the two derivatives as coinciding. For a better comparison with Section 2 it is useful to formulate the dynamics in terms of utilization rather than $a$. To this end, we note that by differentiating (4) with respect to time we get $\dot{a}=(s \pi-\gamma) \dot{u}$. With $g_{y}-a=\hat{u}+g_{k}-a=\hat{u}+a+\gamma\left(u-u_{n}\right)-a$, the latter from (2) and (3), equation (15) becomes $(s \pi-\gamma) \dot{u}=\beta\left[\dot{u} / u+\gamma\left(u-u_{n}\right)\right]$. Solving for $\hat{u}$ leads to the differential equation

$$
\hat{u}=\frac{\gamma}{(s \pi-\gamma) u / \beta-1}\left(u-u_{n}\right)
$$

The difference from Shaikh's equation (7) is that the coefficient on the utilization gap varies with $u$. Nevertheless, considering values of $u$ below an arbitrarily high upper bound, the coefficient will always be negative if only $\beta$ is large enough. Hence we have a stable adjustment process again, with Shaikh's dynamics in the limit, $\beta \rightarrow \infty$; and once again we have the contradiction between credibility and a mathematical stability result.

It will nevertheless be noticed that the fraction in (17) is positive for slow adjustments in the sales expectations - that is, for small values of $\beta$ - and it increases and with it the speed at which the utilization rate diverges if $\beta$ rises. This speed tends to infinity as $\beta$ approaches $\widetilde{\beta}:=(s \pi-\gamma) u_{n}$; eventually the dynamics is even undefined when $\beta=\widetilde{\beta}$, and an extremely rapid convergence towards $u_{n}$ is obtained for $\beta$ slightly above $\widetilde{\beta}$. This property of (17) may sow some distrust in the validity of the differential equation with 
a negative fraction. A positive fraction, on the other hand, can make good economic sense: higher values of $\beta$ in (16) should accelerate the positive autofeedback, and we find this reflected as higher values of the (positive) composite coefficient on $u$ in the utilization dynamics (17).

\subsection{Embedding the continuous-time version in a broader framework}

The suspicious forward and backward derivatives in one equation can be avoided if utilization is turned from a statically endogenous variable, $u=u(a)$, into a dynamic variable proper. To this end, we may recall the standard story that is underlying the assumption of continuous goods market clearing. It allows for disequilibrium and assumes a mechanism where the level of output is given in the so-called ultra-short period and firms adjust their production upward or downward in order to reduce the gap between demand and supply. Hein et al. (2011: 590) propose that it is directly capital utilization that responds to excess demand, $\dot{u}=\mu\left(g_{k}^{i}-g^{s}\right)$ for some positive reaction coefficient $\mu>0$. We take up this idea, only that we here assume that these quantity adjustments take place on the same timescale as the updating of the benchmark rate $a$. Let us thus treat utilization as a predetermined variable in time $t$ and, with the savings and investment functions (1) and (2), describe its changes over time by the equation

$$
\dot{u}=\mu\left[a+\gamma\left(u-u_{n}\right)-s \pi u\right] .
$$

The original IS temporary equilibrium framework is recovered in the limiting case of an infinite adjustment speed, so to speak, $\mu \rightarrow \infty$.

It may be mentioned that specification (18) can be derived from a behavioural assumption on the rate of change of the level of production, namely $\dot{Y}=g_{k} Y+\mu\left(g_{k}^{i} K-g^{s} K\right)$. The output growth rate thus reads $g_{y}=\dot{Y} / Y=g_{k}+\mu\left[a+\gamma\left(u-u_{n}\right)-s \pi u\right] / u$, and substituting this in the identity $\hat{u}=g_{y}-g_{k}$ gives rise to (18).

The term $g_{k} Y$ makes good economic sense in these output adjustments if it is taken into account that we have (implicitly) ruled out any rationing of the agents, which implies the existence of inventories. A positive excess demand is then served from the stock of inventories and a positive excess supply is absorbed by it. If firms are not to run out of inventories through these fluctuations in the long run, this stock has to grow with the rest of the economy. The term $g_{k} Y$ can thus be viewed as representing such a (voluntary) inventory investment in a conveniently simple way. ${ }^{7}$

With the output growth rate $g_{y}$ just determined and substituting (2) there for the capital growth rate $g_{k}$, the adaptive expectations equation (15) becomes

$$
\dot{a}=\beta\left\{\mu\left[a+\gamma\left(\mathrm{u}-u_{n}\right)-s \pi u\right] \frac{1}{u}+\gamma\left(u-u_{n}\right)\right\} .
$$

In this way a (slightly non-linear) two-dimensional differential equations system is obtained with the 'animal spirits' growth rate $a$ as well as utilization $u$ as its dynamic state variables. Obviously, $a^{o}=s \pi u_{n}$ and $u^{o}=u_{n}$ is a point of rest for (18) and (19).

7. A more explicit feedback of inventories on production is possible and straightforward to consider; see the model put forward by Franke (1996) that is built along Metzlerian lines in a growth context and is still analytically tractable. We abstain from an extension in this direction because, besides having an inventory ratio as a third state variable, this would introduce additional, possibly destabilizing mechanisms (especially Metzler's so-called inventory accelerator). 
The partial derivatives of the right-hand sides of these equations evaluated at $\left(u^{o}, a^{o}\right)$ and then the trace and determinant of their Jacobian matrix are easily calculated. We get:

$$
\begin{aligned}
\text { trace } J & =-\mu\left(s \pi-\gamma-\beta / u_{n}\right) \\
\operatorname{det} J & =\beta \mu\left[(\mu-1)(s \pi-\gamma) / u_{n}-\gamma\right] .
\end{aligned}
$$

Sufficient and necessary conditions for stability are a negative trace and positive determinant. Given the Keynesian stability, $\gamma<s \pi$, a positive determinant is ensured if the output adjustments are fast enough; that is, if $\mu$ is large enough. Taking this for granted, we arrive at a stability statement that is exactly the opposite of the previous subsection. The trace is positive and therefore the steady state is unstable if, independently of $\mu$, the adaptive expectations are so responsive that $\beta>(s \pi-\gamma) u_{n}$. By contrast, stability becomes possible if these expectations are so slow (rather than fast) that $\beta<(s \pi-\gamma) u_{n}$. Practically, however, this speed would have to be so low that this possibility is of no more than academic interest.

The idea of constructing the present model is that (18) and (19) constitute a welldefined dynamic system without any ambiguities. Moreover, it allows us to view the assumption of continuous goods market clearing as the limiting case $\mu \rightarrow \infty$. As these variations of $\mu$ do not affect the stability conclusion, the markedly different stability results in this and the previous subsection suggest that the stable one-dimensional differential equation for $a$ or $u$ in the latter does not only evoke a feeling of uneasiness but is indeed ill-specified. Unfortunately, however, (18) and (19) do not give rise to a closed-form expression for the changes of one of these variables when we let $\mu$ tend to infinity.

\subsection{Discrete-time modelling}

As in Section 3, the continuous-time results should be checked by reformulating the model in discrete time, within the framework of our $h$-economies. The benchmark rate $a=a_{t}$ of the firms is given at the beginning of period $[t, t+h)$, when $u_{t}$ is not yet observed, and determines the trend in the growth of the capital stock. We have therefore the following dating in the investment function:

$$
g_{k, t+h}^{i}=a_{t}+\gamma\left(u_{t-h}-u_{n}\right) .
$$

We return to the IS assumption, which here again reads $g_{k, t+h}^{i}=g_{t+h}^{s}$. The latter term is given by (11), $g_{t+h}^{s}=s \pi u_{t}$, so that utilization in the present period results in:

$$
u_{t}=\left[a_{t}+\gamma\left(u_{t-h}-u_{n}\right)\right] / s \pi .
$$

Note that the lag in (20) now turns the goods market clearing condition into a dynamic equation. The updating of the benchmark growth rate $a_{t}$ takes place at the end of the period $[t, t+h)$, a time when $Y_{t}$ has already become known. The discretetime counterpart of the adaptive equations in (15) is thus

$$
a_{t+h}=a_{t}+h \beta\left(g_{y, t}-a_{t}\right) .
$$

For the output growth rate, equation (9) with $j=0$ can be used, where we replace $u_{t}$ with (21) and $g_{k, t}$ with $g_{t}^{s}=s \pi u_{t-h}$. This gives us:

$$
a_{t+h}=a_{t}+\beta\left[\frac{a_{t}+\gamma\left(u_{t-h}-u_{n}\right)}{s \pi u_{t-h}}\left(1+b s \pi u_{t-h}\right)-1-h a_{t}\right] .
$$


Equations (21) and (22) set up another non-linear two-dimensional difference equations system for $u_{t}$ and $a_{t}$. Once again, however, no well-defined continuous-time limit for $h \rightarrow 0$ exists. $^{8}$

As before, a steady-state position is easily seen to be given by $u^{o}=u_{n}$ and $a^{o}=s \pi u_{n}$. The $2 \times 2$ Jacobian matrix of (21) and (22) evaluated at this point is

$$
J=\left[\begin{array}{cl}
\gamma / s \pi & 1 / s \pi \\
-\beta\left[(s \pi-\gamma) / a^{o}-b \gamma\right] & 1+\beta / a^{o}
\end{array}\right] .
$$

It gives rise to the following coefficients in the Schur conditions (using $s \pi=a^{o} / u_{n}$ ):

$$
\begin{aligned}
& a_{1}:=-\operatorname{trace} J=-\left(1+\gamma / s \pi+\beta / a^{o}\right) \\
& a_{2}:=\operatorname{det} J=\gamma / s \pi+\beta\left(1-b \gamma u_{n}\right) / a^{o} .
\end{aligned}
$$

One of the necessary conditions for stability is $1+a_{1}+a_{2}>0$. However, what we obtain is:

$$
1+a_{1}+a_{2}=-b \beta \gamma u_{n} / a^{o}<0 .
$$

In the discrete-time setting with continuous IS market clearing the steady state is therefore always unstable, irrespective of the length of the adjustment period $h$, and not even slow adaptive sales expectations are able to stabilize it. The latter is a slight but largely negligible difference from the continuous-time formulation in the previous subsection with adaptive expectations but a dynamic multiplier; that is, quantity adjustments of firms to goods market disequilibrium.

\section{CONCLUSION}

This note was concerned with a recent proposal by A. Shaikh (2009) to solve the fundamental problem of Harrodian instability. His surprisingly simple idea is to adopt the expected growth of sales as the trend rate of growth in the investment function. The positive message of a mathematical stability proposition was, however, not unspoilt since it contradicts a straightforward reasoning on the dynamic feedbacks, which finds no clue to a stabilizing effect. To be precise, this negative result is based on (in some sense) adaptive, or 'backwardlooking', expectations, while stability would (almost trivially) be confirmed with rational, or 'forward-looking', expectations. However, in neo-Kaleckian or more generally postKeynesian theories the latter hypothesis is usually declined, as it is considered to demand too much of the agents in a model. So the puzzle remains.

Shaikh models his idea within a continuous-time framework. A crucial point is that here is an equation with a forward and, with backward-looking firms, a backward derivative. The two are assumed to coincide and so cancel out, such that we are left with one negative feedback of utilization on itself. It is the common-sense argument that makes this procedure somewhat suspicious and asks for a closer examination. To this end, the economy was transformed into discrete time. This approach allowed us, in a first round, to distinguish between perfect foresight, myopic perfect foresight, and static expectations.

8. In the limit $h \rightarrow 0$, equation (21) re-establishes the IS utilization $u_{t}=u\left(a_{t}\right)$ from (4), and (22) collapses into the non-informative statement $0=\beta\left(s \pi u_{t} / s \pi u_{t}-1\right)$. 
Working with an adjustment period of flexible length $h$, Shaikh's stable differential equation was indeed recovered in the first two cases when we let $h$ tend to zero. This finding improves upon the informal reasoning in that we now know that it 'only' takes myopic perfect foresight to achieve stability. Nevertheless, while at first sight this is an assumption that might be similarly acceptable to IS market clearing, we argued that (at least in our discrete-time story) it is more demanding.

The (in this respect) more appropriate model version with static (or naive) expectations proved to be noteworthy for two reasons. First, it has no well-defined limit for $h \rightarrow 0$, and second, the steady state of the discrete-time economy is unstable for all values of $h$. These investigations were subsequently complemented by introducing adaptive expectations in continuous as well as discrete time, which by and large supported the previous findings.

These results and the way in which they were derived provide a rigorous basis to conclude that Shaikh's concept of the, as he calls it, Hicks-Harrod dynamic adjustment principle amounts, willingly or not, to the assumption of (myopic) perfect foresight. By contrast, if one is not willing to accept this hypothesis, the principle baulks at a simple continuous-time modelling, while in the safer discrete-time framework (or under adaptive expectations with goods market disequilibrium) the original destabilizing Harrodian forces continue to be fully effective. In sum, backward-looking expectations or adjustments formulated in an elementary Keynesian or neo-Kaleckian model of the goods market are not able to overturn this basic mechanism.

\section{REFERENCES}

Cellarier, L. (2006): Constant gain learning and business cycles, in: Journal of Macroeconomics, 28, $51-85$.

Evans, G., Honkapohja, S. (2001): Learning and Expectations in Macroeconomics, Princeton, NJ: Princeton University Press.

Flaschel, P., Franke, R., Semmler, W. (1997): Dynamic Macroeconomics: Instability, Fluctuations, and Growth in Monetary Economies, Cambridge, MA: MIT Press.

Foley, D.K. (1975): On two specifications of asset equilibrium in macroeconomic models, in: Journal of Political Economy, 83, 303-324.

Franke, R. (1996): A Metzlerian model of inventory growth cycles, in: Structural Change and Economic Dynamics, 7, 243-262.

Gandolfo, G. (1997): Economic Dynamics, Berlin: Springer.

Hein, E., Lavoie, M., Treeck, T. van (2011): Some instability puzzles in Kaleckian models of growth and distribution: a critical survey, in: Cambridge Journal of Economics, 35, 587-612.

Hicks, J.R. (1985): Methods of Economic Dynamics, Oxford: Clarendon Press.

Sargent, T.J. (1999): The Conquest of American Inflation, Princeton, NJ: Princeton University Press.

Shaikh, A. (2009): Economic policy in a growth context: a classical synthesis of Keynes and Harrod, in: Metroeconomica, 60, 455-494.

Skott, P. (2008): Growth, instability and cycles: Harrodian and Kaleckian models of accumulation and income distribution, Working Paper 2008-12, University of Massachusetts, Amherst. (Published in Setterfield, M. (ed.) (2010), Handbook of Alternative Theories of Economic Growth, Cheltenham, UK and Northampton, MA: Edward Elgar, 108-131.) 